\section{Medical Principles and Practice}

Med Princ Pract 2018;27:562-569

DOI: $10.1159 / 000493392$
Received: February 7, 2018

Accepted: September 2, 2018

Published online: September 2, 2018

\title{
Sauna Bathing and Risk of Psychotic Disorders: A Prospective Cohort Study
}

\author{
Tanjaniina Laukkanen $^{\text {a, } c}$ Jari A. Laukkanen ${ }^{\text {a-c }}$ Setor K. Kunutsor ${ }^{d, e}$ \\ anstitute of Public Health and Clinical Nutrition, University of Eastern Finland, Kuopio, Finland; ${ }^{b}$ Faculty of Sport \\ and Health Sciences, University of Jyväskylä, Jyväskylä, Finland; ' Central Finland Health Care District, Jyväskylä, \\ Finland; ${ }^{\mathrm{d}}$ Translational Health Sciences, Bristol Medical School, University of Bristol, Southmead Hospital, Learning \\ \& Research Building (Level 1), Bristol, UK; ${ }^{~}$ National Institute for Health Research Bristol Biomedical Research Centre, \\ University of Bristol, Bristol, UK
}

\section{Significance of the Study}

- Sauna bathing has numerous health benefits including the promotion of mental well-being and relaxation; however, its association with mental disorders is unknown. In this new study, frequent sauna bathing was strongly associated with a reduced risk of psychotic disorders. These new findings add to emerging evidence that frequent sauna therapy could reduce the risk of several acute and chronic health conditions.

\section{Keywords}

Sauna $\cdot$ Mental health $\cdot$ Psychosis $\cdot$ Risk factor $\cdot$ Cohort study

\section{Abstract}

Objective: Sauna bathing has been suggested to promote mental well-being and relaxation, but the evidence is uncertain with respect to mental disorders. We aimed to assess the association of frequency of sauna bathing with risk of psychosis in the Kuopio Ischemic Heart Disease prospective population-based study. Subjects and Methods: Baseline sauna bathing habits were assessed in 2,138 men aged 4261 years who had no history of psychotic disorders. Participants were classified into three groups based on the frequency of sauna bathing (once, 2-3, and 4-7 times per week). Results: During a median follow-up of 24.9 years, 203 psychotic disorders were recorded. A total of $537,1,417$, and
184 participants reported having a sauna bath once a week, 2-3 times, and 4-7 times per week, respectively. In Cox regression analysis adjusted for age, compared to men who had 1 sauna session per week, the hazard ratio (95\% confidence intervals) of psychosis for 4-7 sauna sessions per week was 0.23 (0.09-0.58). In a multivariable model adjusted for several risk factors and other potential confounders, the corresponding hazard ratio was 0.21 (0.08-0.52). The association was similar after further adjustment for total energy intake, socioeconomic status, physical activity, and C-reactive protein (0.22 [0.09-0.54]) and was unchanged on additional adjustment for duration of a sauna session and temperature of the sauna bath (0.23 [0.09-0.57]). Conclusion: Our study suggests a strong inverse and independent association between frequent sauna bathing and the future risk of psychotic disorders in a general male population.

(C) 2018 The Author(s)

Published by S. Karger AG, Basel

\begin{tabular}{ll}
\hline KARGER & $\begin{array}{l}\text { ( } 2018 \text { The Author(s) } \\
\text { Published by S. Karger AG, Basel }\end{array}$ \\
E-Mail karger@karger.com & $\begin{array}{l}\text { This is an Open Access article licensed under the Creative Commons } \\
\text { Attribution-NonCommercial-4.0 International License (CC BY-NC) } \\
\text { (http://www.karger.com/Services/OpenAccessLicense), applicable to } \\
\text { the online version of the article only. Usage and distribution for com- } \\
\text { mercial purposes requires written permission. }\end{array}$
\end{tabular}

Jari Laukkanen

Institute of Public Health and Clinical Nutrition

University of Eastern Finland

PO Box 1627, FI-70211 Kuopio (Finland)

E-Mail jariantero.laukkanen@uef.fi 


\section{Introduction}

Psychosis is triggered by other conditions such as schizophrenia, schizoaffective disorders, delusional disorders, neurodegenerative disorders, and major depressive disorders. Psychotic disorders are serious mental disorders, with a lifetime prevalence of approximately $3 \%$ in the general population [1]. Although a relatively rare condition, psychosis is a severe disabling sickness and associated with high health costs and reduced life expectancy [2]. Though very little is known about its etiology, psychosis is regarded as a multifactorial disorder which is determined by genetic and biological factors as well as environmental stressors [3]. Factors associated with low socioeconomic status (SES) such as low education and income levels, unemployment, and being unmarried, widowed, or divorced have been found to be linked to an increased risk of psychotic disorders [4-6]. Compelling evidence also suggests that psychosocial factors such as stress, depression, and anxiety are strongly implicated in the development of psychotic disorders [7-10]. Evidence suggests that these factors act in a cumulative way to increase the risk of psychosis [11]. Psychosis is also a very common feature in people suffering from Alzheimer's disease [12].

Sauna bathing, an activity linked to relaxation and well-being, which is commonly used and readily available in Nordic countries especially [13], has been linked with numerous health benefits which include improvement in the pain and symptoms associated with musculoskeletal diseases, treatment of chronic headache and reduced risk of respiratory diseases, hypertension, cardiovascular disease, stroke, and mortality [14-16]. Frequent sauna bathing has also been shown to be associated with a reduced risk of dementia and Alzheimer's disease [17] and reduces the symptoms of depression [18]. Other passive heat therapies such as Waon therapy, infrared saunas, and whole-body hyperthermia have also been shown to relieve stress and improve the symptoms of psychiatric disorders such as depression and anxiety [18-20].

Given the strong relationship between psychotic disorders and factors such as stress, depression, anxiety, and dementia, as well as emerging evidence on the beneficial effects of sauna bathing or thermal therapy on these factors or outcomes, we hypothesized that sauna bathing may be linked to improved mental health. In this context, we aimed to assess the association of frequency of sauna bathing with the risk of psychosis, using a populationbased cohort of 2,138 men who were free from any apparent mental illness at baseline.

Sauna Bathing and Psychosis

\section{Methods}

\section{Study Design and Participants}

The study population comprised a randomly selected representative sample of middle-aged men aged 42-61 years who were recruited into the Finnish Kuopio Ischemic Heart Disease (KIHD) risk factor study. Details of recruitment methods, ethical permissions, and assessment of risk markers and sauna bathing have been described in a previous report [16]. Briefly, subjects were a randomly selected sample of 3,433 men who resided in the town of Kuopio or its surrounding rural communities. Of those invited, 2,682 volunteered to participate in the study and those with complete information on sauna bathing were included $(n=2,327)$. Baseline examinations were conducted between March 1984 and December 1989. Only 12 men did not use sauna at all and were excluded from the analysis. Men with a history of use of antipsychotic medication were excluded at baseline $(n=52)$. The final dataset analyzed comprised 2,138 men with complete information on sauna exposure, relevant covariates, and psychosis outcome. The investigation was concordant with the principles outlined in the Declaration of Helsinki and its future amendments. The study protocol was approved by the Research Ethics Committee of the University of Eastern Finland and each participant gave written informed consent.

\section{Assessment of Covariates and Exposure}

Participants were instructed to fast overnight, abstain from alcohol consumption for at least 3 days, and to keep away from smoking for at least $12 \mathrm{~h}$ prior to blood specimen collections. The cholesterol contents of serum lipoprotein fractions and triglycerides were measured enzymatically (Boehringer Mannheim, Germany) [16]. High-sensitivity serum C-reactive protein was assessed with an immunometric assay (Immulite High Sensitivity C-Reactive Protein Assay; DPC, Los Angeles, CA, USA). Participants completed a self-administered health and lifestyle questionnaire for the assessment of age, smoking, alcohol consumption, level of physical activity, SES, baseline diseases, and medication history. The energy expenditure of physical activity was assessed using the validated KIHD 12-month leisure time physical activity history questionnaire [14]. Adulthood SES was assessed as a combined measure of income, education, occupation, occupational prestige, material standard of living, and housing conditions, all of which were assessed with self-reported questionnaires. Values for SES ranged from 0 to 25, with a higher value indicating lower levels of SES. Alcohol consumption was assessed using the Nordic Alcohol Consumption Inventory. History of type 2 diabetes was defined as having a clinical diagnosis of diabetes and regular treatment with diet, oral hypoglycemic agents or insulin therapy, fasting plasma glucose $\geq 7.0$ $\mathrm{mmol} / \mathrm{L}$, or according to self-reports. History of coronary heart disease (CHD) was based on a previous myocardial infarction, angina pectoris, the use of nitroglycerin for chest pain once a week or more frequently, or chest pain. Resting blood pressure was measured between 8 and 10 a.m. with a random zero sphygmomanometer. The weekly frequency of sauna bathing sessions, duration of sessions and temperature of the sauna room were assessed using self-administrated questionnaires. The assessment represented typical sauna bathing habits during the week. The questionnaires were checked by an experienced nurse at the time of baseline examination. 
Table 1. Baseline characteristics of overall study participants and according to frequency of sauna bathing

\begin{tabular}{|c|c|c|c|c|c|}
\hline \multirow[t]{2}{*}{ Characteristic } & \multicolumn{5}{|c|}{ Sauna bathing (sessions per week) } \\
\hline & $\begin{array}{l}\text { overall } \\
(n=2,138)\end{array}$ & $\begin{array}{l}1 \\
(n=537)\end{array}$ & $\begin{array}{l}2-3 \\
(n=1,417)\end{array}$ & $\begin{array}{l}4-7 \\
(n=184)\end{array}$ & $\begin{array}{l}p \text { value for } \\
\text { heterogeneity }\end{array}$ \\
\hline \multicolumn{6}{|l|}{ Questionnaire/prevalent conditions } \\
\hline Age at survey, years & $53.1(5.1)$ & $53.4(5.0)$ & $53.2(5.0)$ & $51.5(5.7)$ & $<0.001$ \\
\hline Alcohol consumption, g/week & $31.4(6.4-88.4)$ & $32.3(7.2-96.0)$ & $30.1(6.3-84.4)$ & $39.2(8.0-114.0)$ & 0.036 \\
\hline Total energy intake, kJ/day & $9,862(2,534)$ & $9,559(2,471)$ & $9,898(2,532)$ & $10,474(2,621)$ & $<0.001$ \\
\hline Socioeconomic status & $8.29(4.24)$ & $8.45(4.27)$ & $8.31(4.21)$ & $7.63(4.26)$ & 0.071 \\
\hline History of diabetes & $76(3.6)$ & $26(4.8)$ & $48(3.4)$ & $2(1.1)$ & 0.05 \\
\hline Current smokers & $647(30.3)$ & $190(35.4)$ & $424(29.9)$ & $33(17.9)$ & $<0.01$ \\
\hline History of CHD & $510(23.9)$ & $134(25.0)$ & $343(24.2)$ & $33(17.9)$ & 0.135 \\
\hline \multicolumn{6}{|l|}{ Physical measurements } \\
\hline BMI & $26.8(3.5)$ & $26.8(3.5)$ & $26.7(3.4)$ & $27.9(4.2)$ & $<0.001$ \\
\hline SBP, mm Hg & $134(17)$ & $135(18)$ & $133(16)$ & $135(17)$ & 0.095 \\
\hline $\mathrm{DBP}, \mathrm{mm} \mathrm{Hg}$ & $88(10)$ & $89(11)$ & $88(10)$ & $90(12)$ & 0.093 \\
\hline Physical activity, kJ/day & $1,223(662-2,022)$ & $1,136(568-1,830)$ & $1,238(682-2,038)$ & $1,510(701-2,362)$ & 0.003 \\
\hline \multicolumn{6}{|l|}{ Lipid markers } \\
\hline Total cholesterol, mmol/L & $5.90(1.07)$ & $5.87(1.11)$ & $5.93(1.06)$ & $5.79(0.97)$ & 0.209 \\
\hline $\mathrm{HDL}-\mathrm{C}, \mathrm{mmol} / \mathrm{L}$ & $1.30(0.30)$ & $1.27(0.28)$ & $1.31(0.31)$ & $1.33(0.31)$ & 0.021 \\
\hline Triglycerides, mmol/L & $1.11(0.81-1.57)$ & $1.13(0.79-1.60)$ & $1.10(0.81-1.55)$ & $1.10(0.78-1.61)$ & 0.916 \\
\hline \multicolumn{6}{|c|}{ Metabolic and inflammatory markers } \\
\hline Fasting plasma glucose, $\mathrm{mmol} / \mathrm{L}$ & $5.32(1.15)$ & $5.43(1.41)$ & $5.27(1.05)$ & $5.31(1.09)$ & 0.026 \\
\hline Serum creatinine, $\mu \mathrm{mol} / \mathrm{L}$ & $89.6(13.7)$ & $88.7(14.9)$ & $89.6(12.7)$ & $92.1(16.5)$ & 0.02 \\
\hline High-sensitivity CRP, mg/L & $1.24(0.69-2.34)$ & $1.46(0.71-2.85)$ & $1.19(0.67-2.25)$ & $1.21(0.75-2.09)$ & $<0.001$ \\
\hline
\end{tabular}

Values are mean (SD) or n (\%) or median (IQR). BMI, body mass index; CHD, coronary heart disease; CRP, C-reactive protein; DBP, diastolic blood pressure; HDL-C, high-density lipoprotein cholesterol; IQR, interquartile range; SD, standard deviation; SBP, systolic blood pressure.

\section{Ascertainment of Outcomes}

We included all psychotic disorders that occurred from study enrollment through to 2013. Data on hospitalization due to a psychotic disorder were ascertained by computer linkage to the $\mathrm{Na}$ tional Hospital Discharge Register. All participants (using Finnish personal identification codes) are under continuous annual monitoring for incident psychotic cases and cardiometabolic outcomes, including incident cases. An independent committee of researchers reviewed all potential cases of psychosis to obtain a consensus on the diagnosis. Diagnoses of psychotic disorders were made according to ICD-9 (290-299) and ICD-10 (F00-F09 and F20-F29) codes. Follow-up for incidence of psychotic disorders was conducted using record linkage with the files of the population-based countrywide Finnish Hospital Registry.

\section{Statistical Analysis}

Descriptive data are presented as means (standard deviation, $\mathrm{SD}$ ) or medians (interquartile range, IQR) for continuous variables and percentages for categorical variables. Hazard ratios (HRs) with 95\% confidence intervals (CIs) for psychosis were calculated using Cox proportional hazard models, after confirmation of the assumptions of the proportionality of hazards. Participants were classified into three groups based on the frequency of sauna bathing $(1,2-3$, and 4-7 times per week), to maintain consistency with previous reports $[15,16]$. HRs were progressively adjusted for (i) age (model 1); (ii) plus body mass index (BMI), smoking status, history of diabetes and CHD, years of education, total cholesterol, and alcohol consumption (model 2); (iii) plus total energy intake, SES, physical activity, and high-sensitivity serum C-reactive protein (model 3); and (iv) plus duration of a sauna session and temperature of sauna bath (model 4). Covariates were selected based on their potential as confounders as a result of their known associations with outcomes and observed associations with the exposure using the available data and evidence from previous research. A $p$ value $<0.05$ was considered statistically significant. Statistical analyses were performed using Stata version 14 (Stata Corp., College Station, TX, USA).

\section{Results}

\section{Baseline Characteristics}

A summary of baseline characteristics of overall study participants and according to the group of weekly frequency of sauna bathing is shown in Table 1. The mean 
Fig. 1. Cumulative Kaplan-Meier curves for psychosis during follow-up according to the frequency of sauna bathing per week.

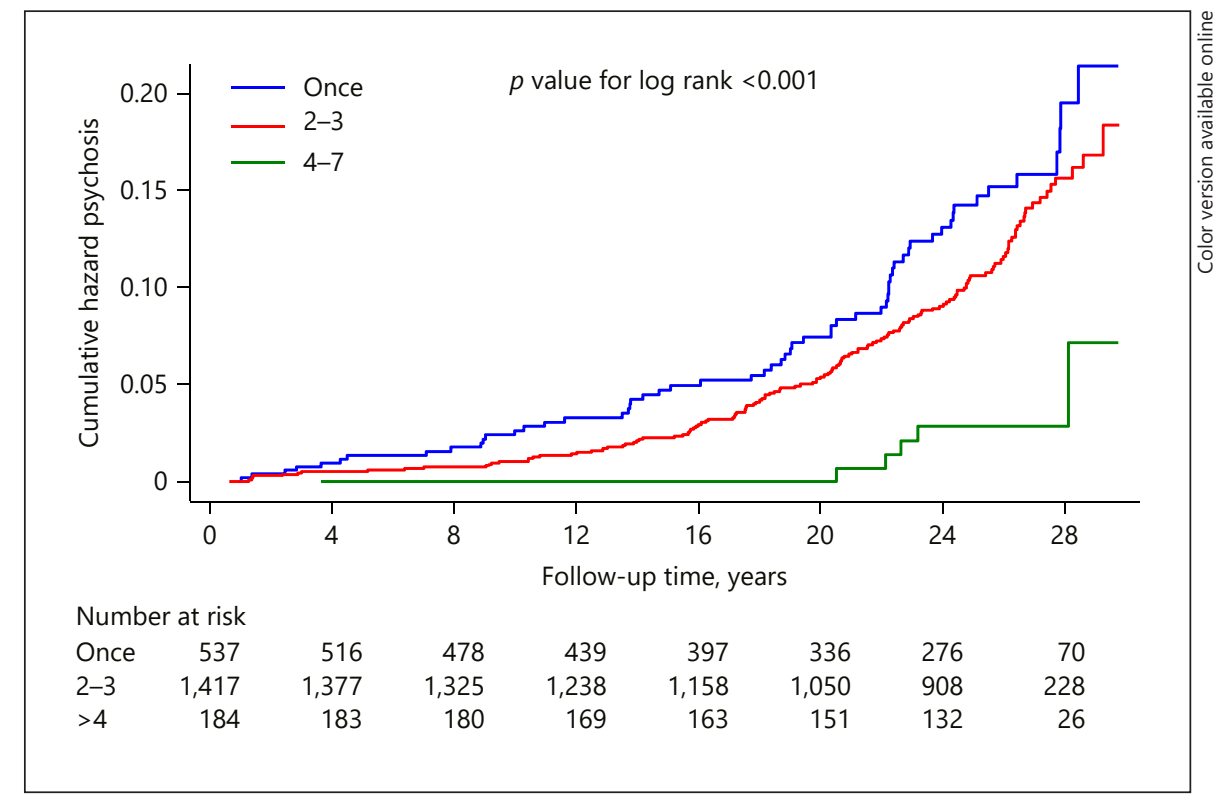

(SD) age was 53.1 (SD, 5.1) years. The median (IQR) frequency of sauna baths and duration of a single sauna session was 2 sessions/week (IQR, 1-3) and 15 min (IQR, 10-15), respectively, with the mean temperature being $79.0^{\circ} \mathrm{C}(\mathrm{SD}, 9.6)$. Participants with the highest frequency of sauna bathing of 4-7 times per week were younger, had higher BMI, lower SES, were more physically active, and consumed more alcohol compared with the participants in the other groups. In this study population, a total of $537,1,417$, and 184 participants reported having a sauna bath once a week, 2-3 times, and 4-7 times per week, respectively.

\section{Sauna Bathing and Risk of Psychosis}

During a median follow-up of 24.9 years (IQR, 18.527.1), 203 hospital-diagnosed psychotic disorders were recorded. Rates of psychotic disorders per 1,000 personyears of follow-up across the three frequency groups of sauna bathing $(1,2-3$, and 4-7 times per week) were 8.1 (95\% CI 6.1-10.8), 7.4 (95\% CI 6.1-9.0), and 2.8 (95\% CI 1.4-5.7), respectively. Cumulative hazard curves demonstrated a lower risk of a psychotic disorder among men who took 4-7 sauna bathing sessions per week compared to the other two groups ( $p<0.001$ for log rank test; Fig. 1 ). Compared to men who had only 1 sauna session per week, the age-adjusted HR (95\% CI) for psychosis was 0.23 ( $95 \%$ CI 0.09-0.58) for men with 4-7 sauna bathing sessions per week. In further analysis adjusted for several conventional risk factors (age, BMI, smoking status, history of diabe- tes and CHD, years of education, total cholesterol, and alcohol consumption), the corresponding association was 0.21 (95\% CI 0.08-0.52). The association was minimally attenuated on additional adjustment for other potential confounders and remained the same on further adjustment for duration of a single sauna session and temperature of the sauna bath $(0.23$ [95\% CI 0.09-0.57]) (Table 2). In further analysis that additionally excluded men who were on antidepressants $(n=19)$, the corresponding adjusted HRs for men who took 4-7 sauna bathing sessions per week (compared to 1 sauna session per week) were 0.24 (95\% CI 0.09-0.59), 0.21 (95\% CI 0.08-0.53), 0.22 (95\% CI 0.09-0.55), and 0.23 (95\% CI 0.09-0.58), respectively. The association between frequency of sauna bathing and risk of psychosis was not significantly modified by any clinically relevant characteristic (Fig. 2).

\section{Discussion}

In this prospective evaluation of the association between frequency of sauna bathing and risk of psychosis, our results showed a strong inverse association between frequency of sauna bathing and future risk of psychosis in men with no previous history of mental illness at baseline. The association remained consistent after excluding men who were taking antidepressants. The association was independent of several potential confounders and not modified by clinically relevant characteristics. 


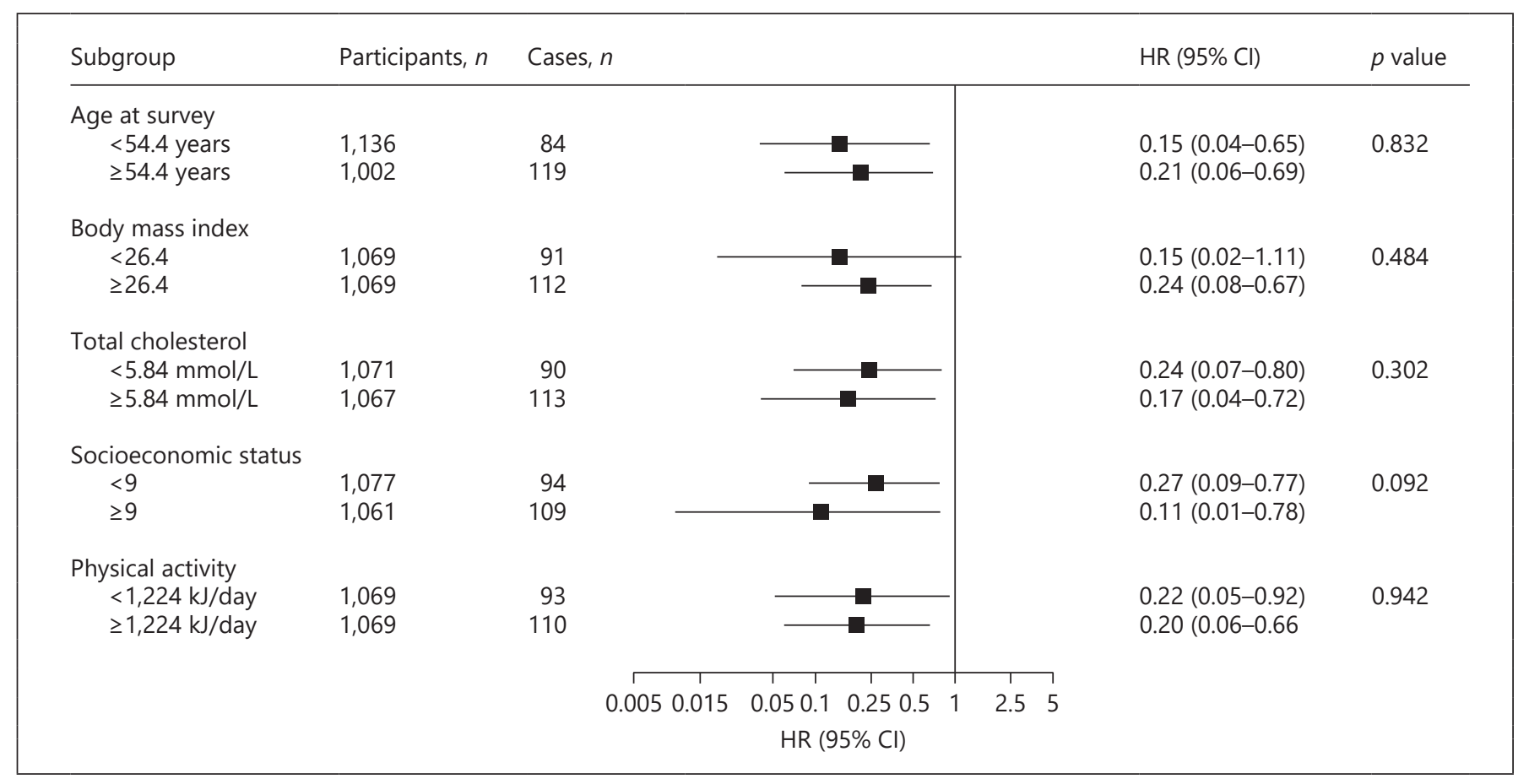

Fig. 2. Association of frequency of sauna bathing with psychosis in clinically relevant subgroups. CI, confidence interval; HDL-C, high-density lipoprotein cholesterol; HR, hazard ratio; HRs are adjusted for age, body mass index, smoking status, history of diabe- tes, prevalent coronary heart disease, years of education, total cholesterol, and alcohol consumption; hazard ratios are reported comparing 4-7 sauna sessions per week with 1 sauna session per week; $* p$ value for interaction; cutoffs used are median values.

Table 2. Association of frequency of sauna bathing and risk of psychosis

\begin{tabular}{|c|c|c|c|c|c|c|c|c|}
\hline \multirow{2}{*}{$\begin{array}{l}\text { Frequency of sauna } \\
\text { bathing (sessions } \\
\text { per week) }\end{array}$} & \multicolumn{2}{|l|}{ Model 1} & \multicolumn{2}{|l|}{ Model 2} & \multicolumn{2}{|l|}{ Model 3} & \multicolumn{2}{|l|}{ Model 4} \\
\hline & HR (95\% CI) & $\begin{array}{l}p \\
\text { value }\end{array}$ & HR (95\% CI) & $\begin{array}{l}p \\
\text { value }\end{array}$ & HR $(95 \%$ CI $)$ & $\begin{array}{l}p \\
\text { value }\end{array}$ & HR (95\% CI) & $\begin{array}{l}p \\
\text { value }\end{array}$ \\
\hline Once & ref & & ref & & ref & & ref & \\
\hline $2-3$ & $0.76(0.56-1.03)$ & 0.082 & $0.78(0.57-1.06)$ & 0.113 & $0.80(0.59-1.09)$ & 0.158 & $0.81(0.59-1.11)$ & 0.190 \\
\hline $4-7$ & $0.23(0.09-0.58)$ & 0.002 & $0.21(0.08-0.52)$ & 0.001 & $0.22(0.09-0.54)$ & 0.001 & $0.23(0.09-0.57)$ & 0.002 \\
\hline
\end{tabular}

CI, confidence interval; HR, hazard ratio; ref, reference; Model 1, adjusted for age; Model 2, model 1 plus body mass index, smoking status, history of diabetes, prevalent coronary heart disease, years of education, total cholesterol, and alcohol consumption; Model 3, model 2 plus total energy intake, socioeconomic status, physical activity, and high-sensitivity C-reactive protein; Model 4, model 3 plus duration of a single sauna session and temperature of sauna bath.

To the best of our knowledge, the prospective association between sauna bathing and the risk of psychotic disorders has not been previously investigated and therefore it is difficult to directly compare our study findings to previous evidence. However, there is evidence to suggest that other types of passive heat therapy such as infrared saunas and Waon therapy improve the mood and reduce depression as well as fatigue, anxiety, and stress $[18,19]$.
A number of studies have also shown that sauna exposure is associated with a reduced risk of several adverse health outcomes [14-16].

The etiology of psychosis is multifactorial and has been reported to result from an interplay between genetic, lifestyle and environmental factors [21]. Although the protective role of sauna bathing in the development of psychosis is not known, we propose several plausible 
mechanistic pathways. Frequent sauna baths enhance relaxation, pleasure and well-being, thereby reducing negative feelings and the anxieties and stresses of everyday life, which are also triggers for psychotic symptoms [7-10]. Sauna bathing is also associated with an opportunity to create friendships, which decrease feelings of isolation, a factor implicated in the development of psychosis [22]. There is evidence suggesting a strong link between elevated hypothalamus-pituitary-adrenal activity and psychosis [23]. Patients with psychotic disorders manifest increased hypothalamus-pituitary-adrenal activity, which is characterized by increased levels of circulating hormones such as cortisol (main stress hormone) and adrenocorticotropin [24]. It is known that agents or illnesses (e.g., Cushing's syndrome) that increase the release of cortisol induce or exacerbate psychotic symptoms [23]. Indeed, several studies have shown that sauna bathing induces hormonal changes which include lowering levels of cortisol by as much as $10-40 \%[25,26]$. Oxidative stress is believed to be involved in the pathophysiology of stress disorders such as chronic fatigue syndrome as well as psychotic disorders [27]. Evidence also suggests that antioxidant depletion and increased oxidative stress are related to the extent of symptoms in chronic fatigue syndrome [28]. Repeated sauna or passive heat therapy reduces levels of markers of oxidative stress [29] and may protect against psychosis via reduction in oxidative stress.

Our findings from hot Finnish sauna bathing with an average temperature of $79^{\circ} \mathrm{C}$ cannot be directly applied to other types of passive heat therapy such as steam rooms, warm water therapy, Waon therapy, and infrared saunas which may operate at lower temperatures; however, similar pathways may be involved. We could not ascertain as to whether the protective effect of sauna on psychotic symptoms is due to the time spent in the hot room, the leisure of a life that allows for more relaxation time, the camaraderie of the sauna, or other biological mechanisms; however, previous evidence in addition to the current findings suggest that sauna bathing, in addition to being a relaxing lifestyle habit, could be used to maintain mental health and well-being. The current study adds to the emerging evidence that sauna bathing has a wide range of health benefits beyond its widely accepted use for relaxation and pleasure.

The results should however be interpreted with caution as our analyses focused on all types of psychotic disorders, including those that are mainly due to memory disorders such as Alzheimer's disease and dementia. The view of sauna as an indicator of SES is indeed stronger and more common in other cultures and countries than in Finland. Although we adjusted for SES, it is not possible to completely exclude that sauna is not an indicator of economic, familial, or working traits. These results could in part be explained by divergent educational background, economic facilities, family engagement, or "attention" to lifestyle among people reporting different sauna use. Also, we did not have data on familiarity as regards mental disorders, use of drugs such as cannabis or an indicator of social deprivation, which may be related to risk of psychosis. There is also a possibility that the findings could be due to "reverse causation," whereby some men were already at risk of developing psychosis at study entry and therefore did not have frequent sauna baths. However, we minimized this bias by excluding study participants who were on antipsychotic medications. The results also remained the same in sensitivity analysis that excluded men on antidepressants.

In the interpretations of our findings, these factors should be considered along with the broad and compelling body of evidence on the beneficial physiological changes of sauna bathing. Other limitations of our study include (i) the inability to examine associations in some subgroups such as smoking status, history of prevalent disease such as diabetes or $\mathrm{CHD}$, due to the limited number of cases in some categories of the exposure and (ii) the inability to generalize the results to women, and therefore it is unknown whether the physiological and psychological benefits of frequent sauna bathing are also applicable to women and older individuals. Furthermore, it was not possible to correct for regression dilution bias which may have underestimated the associations demonstrated, as sauna habits during a typical week were assessed only once. Due to potential changes in the health status of participants over the long period of observation, it is possible that sauna bathing habits may also have changed during follow-up; however, any changes may be minimal as sauna habits are fairly stable in this Finnish population. Finally, we were unable to assess the association between sauna bathing and risk of psychosis when comparing people who used sauna versus those who did not use sauna at all, because of the small numbers who did not use sauna at all. Indeed, a majority of Finnish people are accustomed to having sauna baths at least once per week, as it is a tradition [26].

\section{Conclusion}

In this study, frequent sauna bathing is strongly associated with decreased risk of psychosis in a middle-aged male population. The present study adds to emerging ev- 
idence that frequent passive heat therapy such as sauna bathing could reduce the risk of several acute and chronic health conditions. Sauna bathing is a recommendable habit as it is a well-tolerated and safe activity for most healthy people and even in patients with stable cardiac disease [13]. Potential contraindications include acute infections, unstable angina pectoris, recent myocardial infarction, or severe aortic stenosis [13]. In addition, people with schizophrenia may need to be cautious when taking sauna baths, as evidence suggests that such populations may exhibit dysregulation of body temperature [30]. Further well-conducted research work is needed to confirm the current findings and to understand the mechanisms underlying the observed relationship between sauna bathing and psychosis risk.

\section{Acknowledgements}

The authors thank the staff of the Kuopio Research Institute of Exercise Medicine and the Research Institute of Public Health and University of Eastern Finland, Kuopio, Finland for the data collection in the study.

\section{Statement of Ethics}

The study protocol (143/97) has been accepted by the University of Eastern Finland and Research Ethics Committee Hospital District of Northern Savo, Finland.

\section{Disclosure Statement}

The authors have no conflicts of interest to declare.

\section{Funding Sources}

The study was supported by The Finnish Foundation for Cardiovascular Research, Helsinki, Finland. This source had no role in the design and conduct of the study; collection, management, analysis, and interpretation of the data; and preparation, review, or approval of the manuscript. Dr. Setor Kunutsor acknowledges support from the NIHR Biomedical Research Centre at the University Hospitals Bristol NHS Foundation Trust and the University of Bristol. The views expressed in this publication are those of the author(s) and not necessarily those of the NHS, the National Institute for Health Research or the Department of Health.

\section{References}

1 Perala J, Suvisaari J, Saarni SI, et al: Lifetime prevalence of psychotic and bipolar I disorders in a general population. Arch Gen Psychiatry 2007;64:19-28.

2 McGrath J, Saha S, Chant D, et al: Schizophrenia: a concise overview of incidence, prevalence, and mortality. Epidemiol Rev 2008;30: 67-76.

3 Heckers S: Who is at risk for a psychotic disorder? Schizophr Bull 2009;35:847-850.

4 Kessler RC, Birnbaum H, Demler O, et al: The prevalence and correlates of nonaffective psychosis in the National Comorbidity Survey Replication (NCS-R). Biol Psychiatry 2005; 58:668-676.

5 Miettunen J, Lauronen E, Veijola J, et al: Socio-demographic and clinical predictors of occupational status in schizophrenic psychoses - follow-up within the Northern Finland 1966 Birth Cohort. Psychiatry Res 2007;150: 217-225.

6 Honkonen T, Stengård E, Virtanen M, et al: Employment predictors for discharged schizophrenia patients. Soc Psychiatry Psychiatr Epidemiol 2007;42:372-380.

7 Johns LC, Cannon M, Singleton N, et al: Prevalence and correlates of self-reported psychotic symptoms in the British population. $\mathrm{Br}$ J Psychiatry 2004;185:298-305.
8 Wiles NJ, Zammit S, Bebbington P, et al: Selfreported psychotic symptoms in the general population: results from the longitudinal study of the British National Psychiatric Morbidity Survey. Br J Psychiatry 2006; 188:519-526.

9 Johnson JG, Cohen P, Kasen S, et al: Personality disorder traits associated with risk for unipolar depression during middle adulthood. Psychiatry Res 2005;136:113-121.

10 Hanssen MS, Bijl RV, Vollebergh W, et al: Self-reported psychotic experiences in the general population: a valid screening tool for DSM-III-R psychotic disorders? Acta Psychiatr Scand 2003;107:369-377.

11 Cantor-Graae E: The contribution of social factors to the development of schizophrenia: a review of recent findings. Can J Psychiatry 2007;52:277-286.

12 Murray PS, Kumar S, Demichele-Sweet MA, et al: Psychosis in Alzheimer's disease. Biol Psychiatry 2014;75:542-552.

13 Hannuksela ML, Ellahham S: Benefits and risks of sauna bathing. Am J Med 2001;110: 118-126.

14 Kunutsor SK, Khan H, Zaccardi F, et al: Sauna bathing reduces the risk of stroke in Finnish men and women: a prospective cohort study. Neurology 2018;90:e1937-e1944.

15 Laukkanen JA, Laukkanen T, Kunutsor SK: Cardiovascular and other health benefits of sauna bathing: a review of the evidence. Mayo Clin Proc 2018;93:1111-1121.
16 Laukkanen $\mathrm{T}$, Khan $\mathrm{H}$, Zaccardi F, et al: Association between sauna bathing and fatal cardiovascular and all-cause mortality events. JAMA Intern Med 2015;175:542-548.

17 Laukkanen T, Kunutsor S, Kauhanen J, et al: Sauna bathing is inversely associated with dementia and Alzheimer's disease in middleaged Finnish men. Age Ageing 2017;46:245249

18 Masuda A, Nakazato M, Kihara T, et al: Repeated thermal therapy diminishes appetite loss and subjective complaints in mildly depressed patients. Psychosom Med 2005;67: 643-647.

19 Soejima Y, Munemoto T, Masuda A, et al: Effects of Waon therapy on chronic fatigue syndrome: a pilot study. Intern Med 2015;54: 333-338.

20 Janssen CW, Lowry CA, Mehl MR, et al: Whole-body hyperthermia for the treatment of major depressive disorder: a randomized clinical trial. JAMA Psychiatry 2016;73:789795.

21 Tsuang MT, Stone WS, Faraone SV: Genes, environment and schizophrenia. Br J Psychiatry Suppl 2001;40:s18-s24.

22 Li BJ, Liu P, Chu Z, et al: Social isolation induces schizophrenia-like behavior potentially associated with HINT1, NMDA receptor 1, and dopamine receptor 2. Neuroreport 2017; 28:462-469. 
23 Walker E, Mittal V, Tessner K: Stress and the hypothalamic pituitary adrenal axis in the developmental course of schizophrenia. Annu Rev Clin Psychol 2008;4:189-216.

24 Walsh P, Spelman L, Sharifi N, et al: Male patients with paranoid schizophrenia have greater ACTH and cortisol secretion in response to metoclopramide-induced AVP release. Psychoneuroendocrinology 2005;30: 431-437.

25 Leppaluoto J, Huttunen P, Hirvonen J, et al: Endocrine effects of repeated sauna bathing. Acta Physiol Scand 1986;128:467-470.
26 Kukkonen-Harjula K, Oja P, Laustiola K, et al: Haemodynamic and hormonal responses to heat exposure in a Finnish sauna bath. Eur J Appl Physiol Occup Physiol 1989;58:543550.

27 Owe-Larsson B, Ekdahl K, Edbom T, et al: Increased plasma levels of thioredoxin- 1 in patients with first episode psychosis and longterm schizophrenia. Prog Neuropsychopharmacol Biol Psychiatry 2011;35:1117-1121.
28 Kennedy G, Spence VA, McLaren M, et al: Oxidative stress levels are raised in chronic fatigue syndrome and are associated with clinical symptoms. Free Radic Biol Med 2005;39: 584-589.

29 Masuda A, Miyata M, Kihara T, et al: Repeated sauna therapy reduces urinary 8-epi-prostaglandin F(2alpha). Jpn Heart J 2004;45:297303.

30 Chong TW, Castle DJ: Layer upon layer: thermoregulation in schizophrenia. Schizophr Res 2004;69:149-157. 